\title{
Obituary
}

\section{A tribute to Michael S. Neuberger}

$\mathrm{M}$ ichael S. Neuberger, whose contributions to the field of molecular biology of B lymphocytes paved the way for humanizing therapeutic antibodies and understanding the genetic mechanisms of antibody expression, affinity maturation, and class switching, died October 26th, 2013, of multiple myeloma, at the age of 59 (Figure 1).

Michael Neuberger was the youngest of four sons of the distinguished biochemist, Albert Neuberger, and his artist wife, Lilian. Albert Neuberger left Germany in 1933, as the Nazis came to power, and settled in London where he became a professor of chemical pathology. Remarkably, Fred Sanger, Albert Neuberger's distinguished student, was the mentor of Cesar Milstein, who in turn, started Michael Neuberger along his own career.

Neuberger studied biochemistry at the University of Cambridge and began his scientific career with Brian Hartley at Imperial College London, working on the evolution of enzymes in bacteria. His doctoral work earned him a research fellowship from Trinity College, Cambridge, where he sought out Milstein's help to enter the new field of molecular immunology. Milstein offered him a position in the Medical Research Council Laboratory of Molecular Biology (LMB) but sent him first to the laboratory of Klaus Rajewsky in Cologne to "learn immunology."

After working in Germany for two years on immunoglobulin class-switched antibody pairs, Neuberger returned to the LMB where he began to study the genetic elements that regulated immunoglobulin gene expression in cells and mice transfected with genomic fragments containing functional immunoglobulin genes. This work led to the identification and characterization of transcriptional enhancers that control antibody gene rearrangement, expression, and hypermutation.

Also during this period, Neuberger collaborated with Greg Winter to develop "humanized" therapeutic antibodies tolerated by human patients and with Marianne Bruggemann to produce genetically modified mice that produce human antibodies in response to immunization. Neuberger's identification of the controlling elements for immunoglobulin genes proved to be an important component in the development of a biotechnology that remains a major focus of the pharmaceutical industry.
What most intrigued Neuberger, however, was the unique genetic plasticity of B cells. In addition to the genomic rearrangements of variable $(\mathrm{V})$, diversity $(\mathrm{D})$, and joining $(\mathrm{J})$ gene segments that produce the antibody molecules that act as their antigen receptors, $\mathrm{B}$ cells are also capable of additional genomic rearrangements that alter antibody isotype and can acquire mutations in antibody genes at extraordinary rates. These muta-

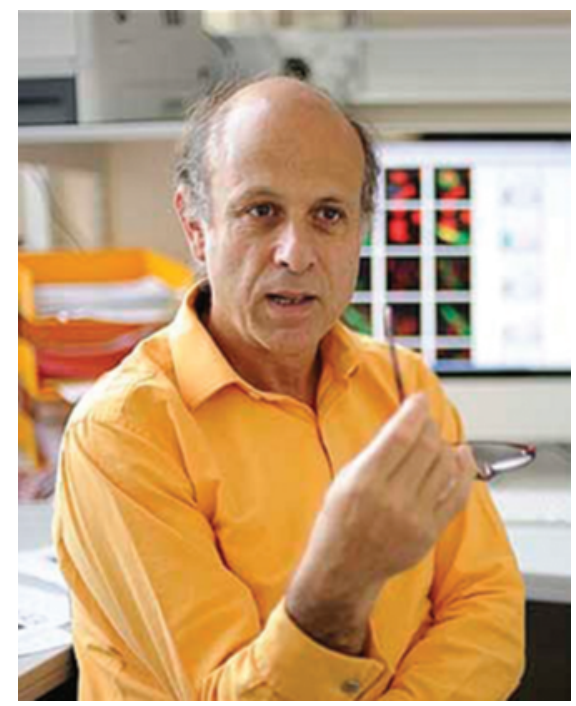

Figure 1

Michael S. Neuberger, Deputy Director, Medical Research Council Laboratory of Molecular Biology, Cambridge, United Kingdom.

tions drive the affinity maturation of antibody and are crucial for effective humoral immunity. Understanding these processes of class-switch recombination and somatic hypermutation became Neuberger's focus.

Neuberger's elucidation of these classes of antibody diversity began in a collaboration with Milstein to create transgenic mice as tools to define the role of promoters and enhancers in the somatic hypermutation of antibody gene loci. Although interpretation of this work was confounded by the random integration of transgenes, Neuberger capitalized on methodological changes in studying antibody gene mutation developed by Milstein that became standard for the field: first, he scored only mutations in the introns downstream of rearranged $\mathrm{V}(\mathrm{D}) \mathrm{J}$ gene segments, and second, he focused his studies on Peyer's patch B cells activated by diverse antigens present in the flora of the small intestine. The first innovation minimized any influence of antigen-driven selection of mutants and the second avoided any requirement for specific immunization and supported the accumulation of very large mutation data sets. In this way, Neuberger identified a DNA motif, WRCY, that strongly favored mutagenesis and was responsible for mutational hot spots in antibody genes. By 1998, Milstein, Cristina Rada, and Neuberger noticed that certain genetic models could separate intronic mutations into discrete phases: an agent that caused G: $\mathrm{C}$ mutations and one that caused A:T mutations.

The first step toward solving the mystery of antibody hypermutation and class switching came with the discovery of an enzyme essential for both activities in mice and humans, activation-induced cytidine deaminase (AID), by Tasuku Honjo and Anne Durandy. Whereas AID was first proposed to function as an RNA editing enzyme (based on its homology to APOBEC1), with considerable insight, Neuberger presented a novel hypothesis based on his previous studies of mutational spectra and AID's enzymatic activity as a cytosine deaminase. AID-mediated deamination of cytosine bases would produce uracils along the transcribed DNA strands encoding the antigen-binding domains [the rearranged V(D)J gene segments] of antibody. He wrote, "It became clear to me that such a mechanism could readily explain that transition/transversion ratios could be shifted according to whether the DNA synthesis occurs opposite a dU residue created by deamination of $\mathrm{dC}$ or whether it occurred over an abasic site generated following uracil DNA glycosylase mediated dU excision" (1). In a stunning trio of papers (2-4) published in 2002, Neuberger proved the DNA deamination model using genetics in bacteria, cell lines, and mice. As shown in an iconic diagram (Figure 2), his hypothesis beautifully explained a common mechanism, not only for antibody hypermutation and class-switch recombination, but also for the diversification of antibodies in birds by gene conversion. It all fell into place. His discovery was as important to understanding the biology of antibodies and $\mathrm{B}$ cells as was the earlier identification of the recombination activating genes that 


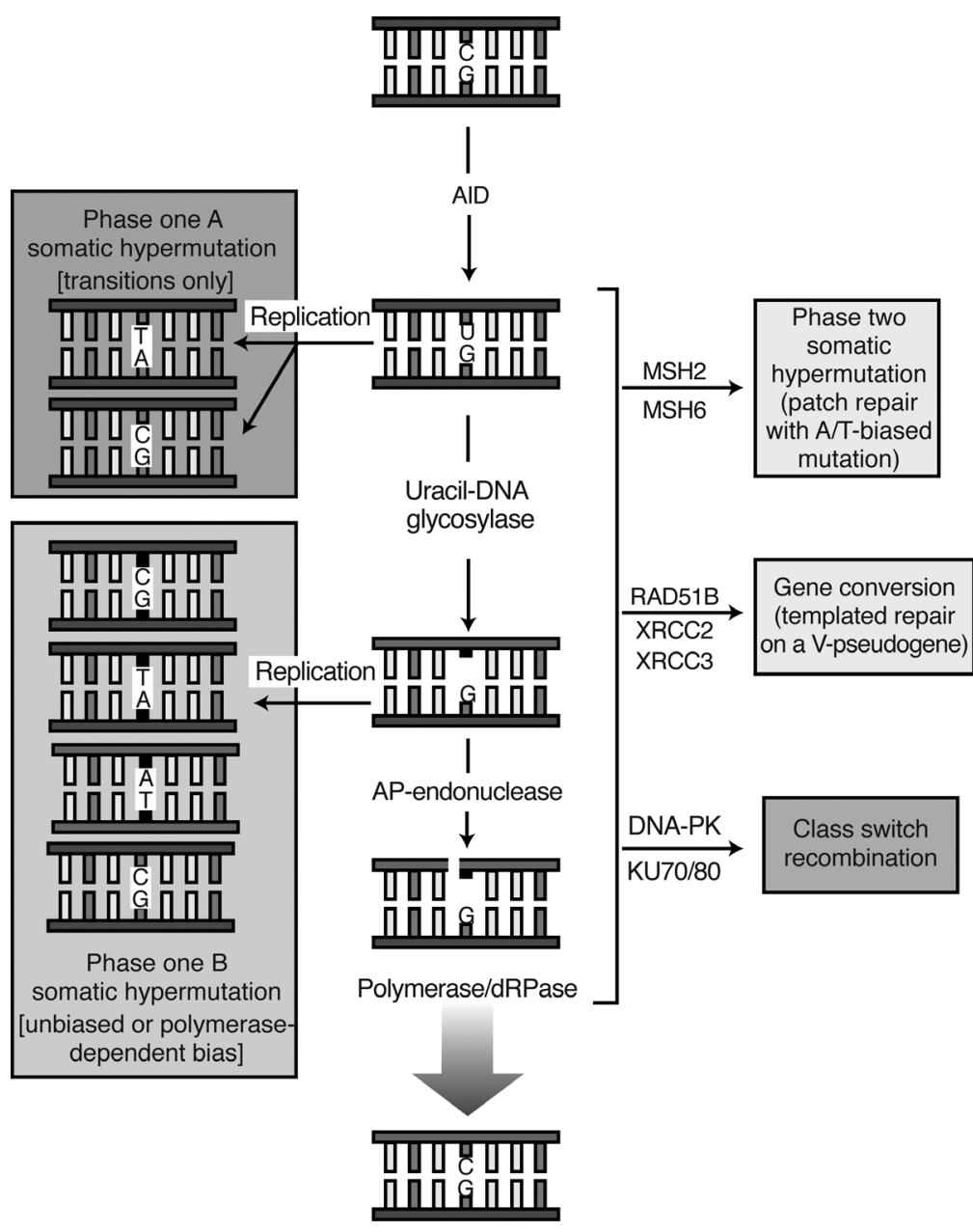

initiated the genomic rearrangement of $\mathrm{V}, \mathrm{D}$, and J gene segments.

These activities of AID were later confirmed by a number of other laboratories, and Neuberger's proposed central mechanism for AID spawned a flurry of papers that expanded on and refined the role of AID in concert with base excision and mismatch repair proteins and low-fidelity DNA polymerases. The agent of G:C mutations recognized initially by Neuberger in antibody genes is the deamination of cytosines by AID, and the A:T mutations are caused by error-prone repair at the U:G lesion by DNA polymerase $\eta(5)$. In both processes, DNA deamination acts as the cornerstone of antibody diversification. The only remaining mystery of this process is how AID is preferentially targeted to immunoglobulin genes.

In another stroke of great insight, Neuberger studied the larger family of APOBEC homologs to investigate the role of other DNA deaminases in mutagenesis. In 2003, he demonstrated that the APOBEC3G enzyme deaminates cytosine in the HIV-1 provirus and inactivates it through hypermutation; subsequently, Neuberger, Rada, and Mike Stratton identified other APOBEC3 proteins that cause hypermutation in breast cancer cells. In retrospect, Neuberger noted that "the deamination of cytosine to uracil itself is an ancient lesion, occurring spontaneously at a rate of 100 to 500 events/cell/day. AID and the APOBEC family of enzymes have merely evolved to selectively accelerate a process that is omnipresent in nature" (6).

Neuberger's unassuming nature and modesty could not conceal the importance of his scientific work. His many honors included appointment as an International Research Scholar of the Howard Hughes Medical Institute (1992), election as a Fellow of the Royal Society (1993), appointment as a Founder Fellow of the Academy of Medical Sciences (2000), the Novartis Medal (2001), the William Bate Hardy Prize (2001), the Prix Jean-Pierre Lecocq (2002), the GlaxoSmithKline Medal (2003), the Dannie-Heineman Prize (2003), and, in 2013, election as a
Figure 2

The standard model for AID-mediated immunoglobulin gene diversification from the seminal work of the Neuberger laboratory (2). The formation of point mutations in the variable region of antibody genes, templated insertion of DNA sequence (gene conversion), and immunoglobulin class switching are all outcomes of a single basic process. AID catalyzes the deamination of cytosine to uracil, followed by removal of the uracil base by uracil-DNA glycosylase and repair by mismatch-repair enzymes and DNA polymerase $\eta$, homologous recombination with a nonfunctional $V$ gene segment (V-pseudogene), or class-switch recombination mediated by DNA-dependent protein kinase (DNA-PK) and KU70/80. AP, apyrimidinic; dRPase, deoxyribophosphodiesterase; $\mathrm{MSH} 2$, mutS homolog 2, mismatch repair enzyme; MSH6, mutS homolog 6, mismatch repair enzyme; RAD51B, homologous recombination repair protein; XRCC2, X-ray repair cross-complementing group 2 , homologous recombination repair protein; XRCC3, X-ray repair cross-complementing group 3 , homologous recombination repair protein. Figure reproduced with permission from Nature.
Foreign Associate of US National Academy of Sciences. In 2002, Neuberger was appointed professor of molecular immunology at the University of Cambridge; Albert Neuberger, elected to the Royal Society in 1951, was able to enjoy his son's election in 1993.

Michael Neuberger's intellectual contributions to others was mostly though his razor sharp ability to get to the core of scientific questions and the fact that he enjoyed talking about science. His influence was magnified by an unassuming and warm personality and his passion for learning from others. He was very generous with his time, had a gentle and self-deprecating sense of humor, and a relaxed attitude to dressing; qualities that endeared him to all.

Neuberger's brothers James, Anthony, and David survive him; James is a professor of medicine at the University of Birmingham, Anthony is a professor of finance at Warwick University, and David, a barrister, is president of the Supreme Court of the United Kingdom. Michael Neuberger leaves his wife 
Gillian, daughters Saskia and Lydia, and sons Thomas and Benjamin. Michael Neuberger was a great and good friend to many, a scientific colleague of the first rank, and a good and decent man. His death is a loss to science, to scholarship and medicine, and to us all.

\section{Patricia J. Gearhart}

Laboratory of Molecular Biology and Immunology, National Institute on Aging, NIH, Baltimore, Maryland, USA. E-mail: gearhartp@mail.nih.gov.

\section{Garnett Kelsoe}

Department of Immunology and Human Vaccine Institute, Duke University, Durham, North Carolina, USA. E-mail: ghkelsoe@duke.edu.

1. Neuberger MS. Cesar Milstein and investigations into the generation of antibody diversity. Immunologia. 2003;22(3):306-312.

2. Petersen-Mahrt SK, Harris RS, Neuberger MS. AID mutates E. coli suggesting a DNA deamination mechanism for antibody diversification. Nature. 2002;418(6893):99-103.

3. Di Noia J, Neuberger MS. Altering the pathway of immunoglobulin hypermutation by inhibiting uracil-DNA glycosylase. Nature. 2002;419(6902):43-48.

4. Rada C, Williams GT, Nilsen H, Barnes DE, Lindahl T, Neuberger MS. Immunoglobulin isotype switching is inhibited and somatic hypermutation perturbed in UNG-deficient mice. Curr Biol. 2002; 12(20):1748-1755.

5. Zeng X, Winter DB, Kasmer C, Kraemer KH, Lehmann AR, Gearhart PJ. DNA polymerase eta is an A-T mutator in somatic hypermutation of immunoglobulin variable genes. Nat Immunol. 2001; 2(6):537-541.

6. Ganesh K, Neuberger MS. The relationship between hypothesis and experiment in unveiling the mechanisms of antibody gene diversification. FASEB J. 2011;25(4):1123-1132. 